\title{
Demonstrations with a Position-Sensitive Detector
}

\author{
Peter J. Millet, Department of Physics, Hamilton College, Clinton, NY 13323; pmillet@ hamilton.edu; \\ and Rosette Khosropour, Department of Physics, Union College, Schenectady, NY 12310
}

W: describe a series of demonstrations that can be easily assembled using some commonly available equipment coupled with a relatively inexpensive device known as a position-sensitive detector, PSD for short. ${ }^{1}$ The techniques described here allow for a vivid indication of subtle movements of surfaces and of slight changes in air density such as accompany ultrasonic sound waves.

When a narrow beam of light illuminates the photosensitive strip of the PSD, two photocurrents, each inversely proportional to the distance of the spot from the far ends of the strip, are produced. The difference in photocurrents is amplified and converted to a voltage by a simple circuit (described in the Appendix). For display purposes that output voltage is fed to an oscilloscope. As shown in Fig. 1, the PSD may be illuminated with an ordinary HeNe teaching laboratory laser. Normal beam divergence (without expansion) results in a 4-mm beam width, when the strip is several meters from the laser, that overlaps the 1-mm width of the photosensitive strip. In one application, (Fig. 1a), as the PSD strip is moved parallel to its length, the region illuminated by the laser beam is altered and the balance of the photocurrents collected from its ends will change. Equivalently, the beam can be swept along the length of a motionless PSD, as in Figs. 1b and 1c. In all cases, since the balance of the photocurrents is sensitive to the centroid of the beam spot, the spatial resolution is much better than the width of the spot.

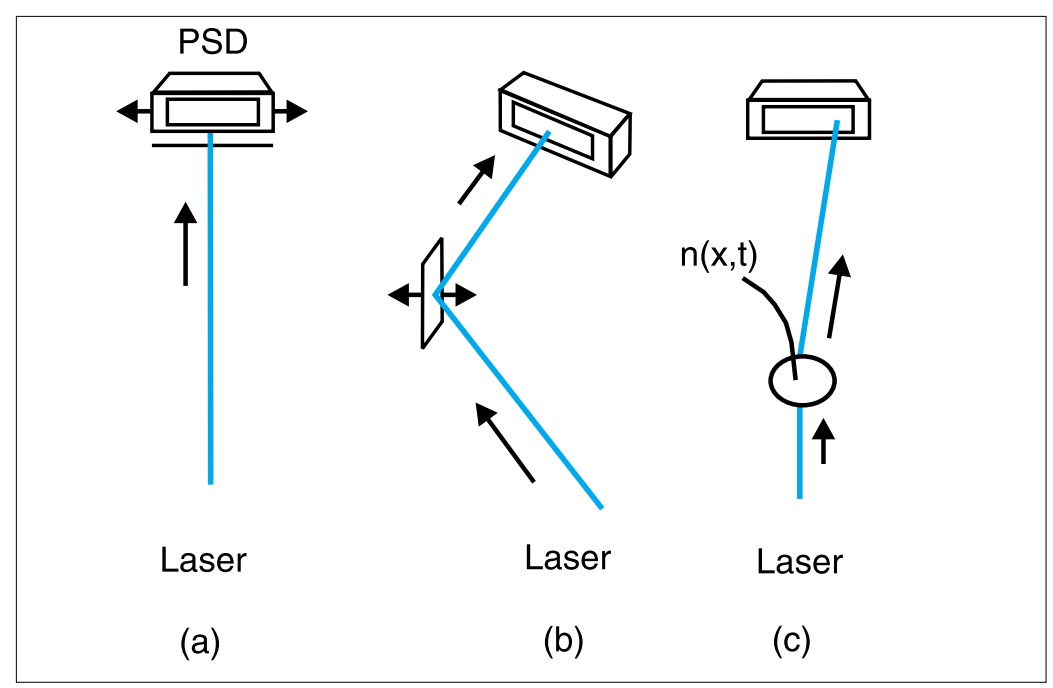

Fig. 1. Three configurations for detecting movement with the PSD: (a) move the PSD; (b) move a surface reflecting the beam onto the PSD; (c) deflect the beam by altering the index of refraction along its path.

According to specifications, the PSD we used has a photosensitive strip of $1-\mathrm{mm}$ width by $12-\mathrm{mm}$ length, a rise time of $3 \mu \mathrm{s}$, and position detection error under 50 microns. For example, we are easily able to resolve static displacements of less than 50 microns. To establish this we mounted the PSD on a micro-translation stage and recorded the minimum discernible shift of the oscilloscope beam as we translated the PSD. The support electronics described at the end of the article provides a dc shift of about $1.5 \mathrm{~V}$ when the PSD is translated $1 \mathrm{~mm}$. In the deflection of a laser beam by an ultrasonic wave emitted by an introductory physics lab motion detector, we see a wave with 150 -mV peak-to-peak amplitude and approximately $48-\mathrm{kHz}$ frequency. Thus, a quite ordinary student oscilloscope is adequate for the uses described here.

We have used the PSD to monitor the motion of a resonant mechanical system, as well as in experiments where the laser beam is deflected by dynamic changes in the refractive index of air. We give examples of such processes below.

\section{Mechanical Resonance of a Loudspeaker}

A loudspeaker will display resonant behavior related to such factors as its moving mass, the restoring force due to the suspension of the cone, its location in the room, and the design of the speaker cabinet. We found that we can display the variation in speaker response to a range of frequencies by fastening a tiny glass cover to the center of a speaker cone with a small amount of rubber cement (any cloth grille or covering having 
been removed from in front of the cone). The laser beam is then pointed at the cover glass so that its (partial) reflection falls on the PSD (Fig. 1b). A sinusoidal voltage source of variable frequency and amplitude is connected to the input of an audio amplifier connected to the loudspeaker. The variation, with frequency, of response of an inexpensive midrange speaker mounted in a sealed box (infinite baffle) is dramatic. Of course, the driving amplitude can be varied and the nonlinear response studied.

Switching from sinusoidal to square-wave excitation of the loudspeaker at a very low frequency, a few $\mathrm{Hz}$ or so, we find that the pulsing of the speaker produces ringing. This effect is clearly demonstrated-we use a storage scope to display and measure the waveform-and it is readily verified that the ringing frequency is the same as the resonant frequency found using sinusoidal excitation. A dividend of the pulsing technique is that the damping can be modeled and calculated.

\section{Visualizing Acoustic Waves}

Enloe et $\mathrm{al}^{2}$ have described an experiment where a fixed PSD can be used to record the motion of the laser beam. In their application a spark discharge in a gas creates a shock wave that is studied through its dynamic alteration of the gas density. The resultant propagating change in the index of refraction deflects the laser beam passing through the shocked region and allows the experimenter to study the time and space dependence of the gas density. The arrangement is represented schematically in Fig. 1c.

We have found that, under the right circumstances, the oscillating variation in air density caused by sound waves produces an oscillating deflection of a HeNe laser beam that can be detected by a PSD and displayed on an oscilloscope. For students to follow an analysis of this sound-light interaction, they must understand how a continuous varia-

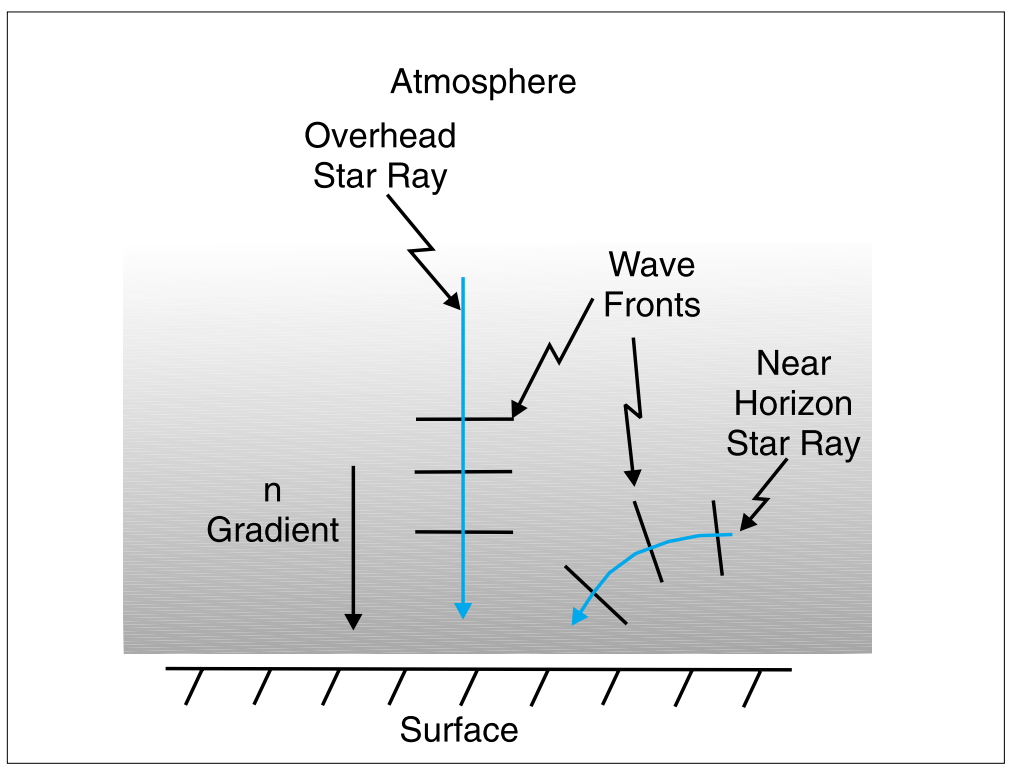

Fig. 2. Deflection of light rays passing through the atmosphere.

tion in index of refraction will affect the path of a ray of light.

A brief but nice qualitative discussion of the continuous case follows Minnaert ${ }^{3}$ in his explanation of the curvature of light rays in the atmosphere, say those from stars, as in Fig. 2 . Thus, we begin by noting that the refractive index of air is higher nearer Earth's surface where the air is denser. When a typical plane wave front from a star seen near the horizon enters the atmosphere, the low altitude part of the wave front will move more slowly than the part at higher altitude. This rotates the wave front, which in turn bends the direction in which it propagates (denoted by the ray). In contrast, the light falling on an observer from a star directly overhead will not be deflected at all since, as the wave front approaches the ground, all parts of it are slowed equally and there is no rotation. Alternatively, we could describe the deflection of a ray as it passes through the atmosphere by appealing to Fermat's principle, i.e., that the path taken by the ray corresponds to an extremum of the optical path length (or time of flight). In the present instance the time is a minimum, and, following Feynman's argument, we rationalize the shape of the light path by saying that "instead of just going in a straight line, it avoids the dense regions where it goes slowly by getting through them at a steeper tilt."

To extend the argument to the sound-light interaction, the atmospheric effect should be described in more general terms: the atmosphere's density depends on position and, therefore, its index of refraction depends on position; of paramount importance is the relationship between the direction in which the ray propagates and the direction in which the index of refraction changes most rapidly.

We may use the term "gradient" to refer to the vector whose magnitude is the rate of change of index of refraction with position, and whose direction is the direction of most rapid increase of the index of refraction. Thus, summarizing Fig. 2, for the atmosphere the gradient of the index of refraction points downward. The ray deflection will be largest when its travel is perpendicular to the gradient; the deflection vanishes when the ray is parallel to the gradient.

A wave of sound in air produces a spatial variation in air density and, thus, in the index of refraction, $n$ (Fig. 3). Moving from density trough to peak to trough, the gradient in $n$ is 


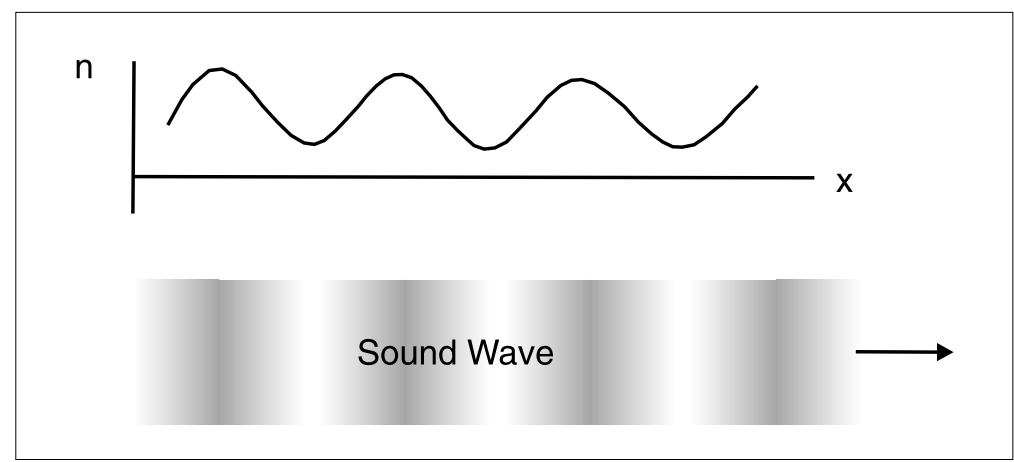

Fig. 3. Variation in index of refraction produced by a sound wave in air. Darker shade represents a higher air density.

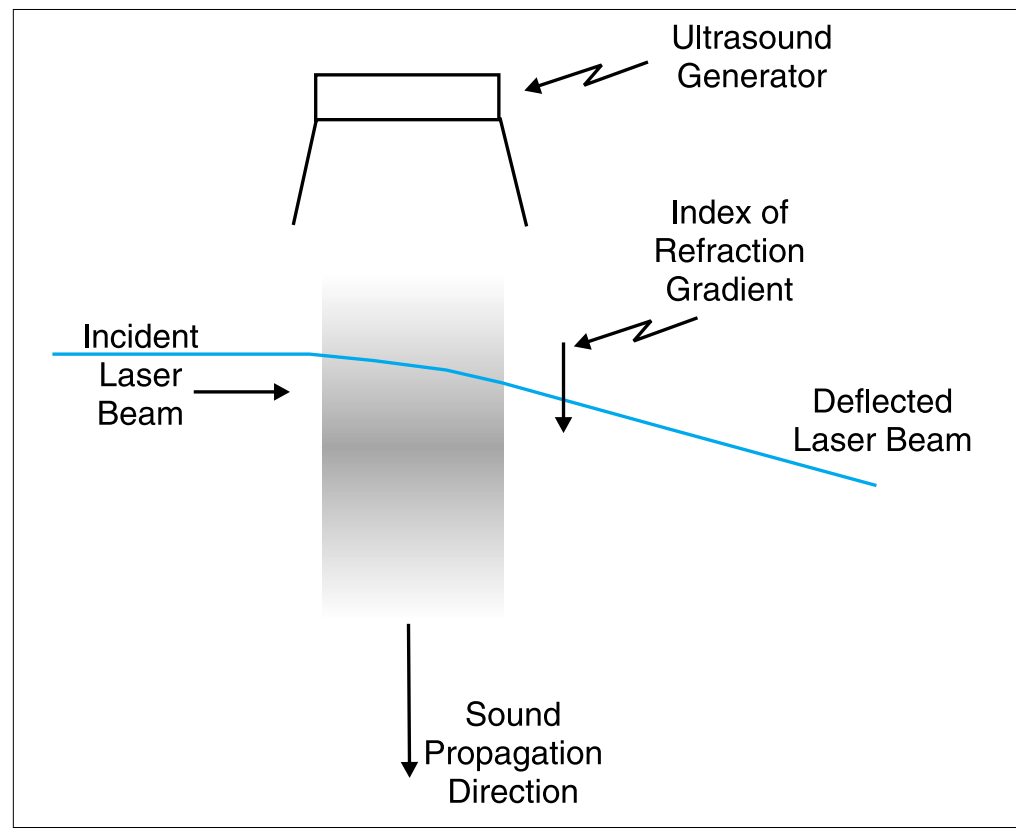

Fig. 4. Momentary deflection of a laser beam passing through a sound wave produced by an ultrasonic motion detector.

alternately parallel and antiparallel to the direction of sound propagation (it is convenient to think in terms of plane waves for both sound and light). As illustrated in Fig. 4 (with greatly exaggerated deflection and wavelength), a light ray initially perpendicular to the direction of propagation of the sound beam will be deflected toward the region of higher index of refraction. Since the direction of the deflection depends on the direction of the gradient, the deflected beam will swing back and forth as the sound wave propagates across the path of the laser beam. The deflection depends on the magnitude of the gra- dient of $n$; so, if $n$ does not vary rapidly enough with spatial coordinate, the effect will be difficult to see. We have found that the effect is clearly visible on an ordinary oscilloscope when an ultrasonic motion detector ${ }^{5}$ is placed so its emission crosses the laser beam as in Fig. 4. The laser beam crosses the ultrasound beam within a few centimeters of the generator and the PSD is several meters from the laser. The distance from the laser to the generator is 10 to $20 \mathrm{~cm}$. On the other hand, when a loudspeaker producing a frequency equal to a few thousand $\mathrm{Hz}$ was used, we found that the relatively long wave- length, taken together with sound levels that were comfortable, produced too low a gradient in $n$ to give a detectable deflection using an oscilloscope alone. ${ }^{6}$

There are a number of nice points to be made with this demonstration. First and foremost is confirmation of the notion that a sound wave dynamically alters the density of the medium in an oscillatory way. It is also immediately apparent that our ultrasound generator produces wave trains or pulses. We can see 16 periods of constant amplitude followed by a ring-down of the transducer 7 or 8 periods long.

We have examined interference between sound waves with this demonstration. To do so we place a piece of plywood in the path of the ultrasound, so that most of the pulse is reflected back toward the source. By arranging the reflector to be close to the laser beam, the superposition of the incoming pulse and its reflection can be examined. Thus, with the reflector positioned so there is a node where the laser crosses the acoustic field, the oscilloscope trace shows oscillation at the beginning and end of a trace with a flat trace in between. This shows that, since the ultrasonic generator produces pulses, the interference will not occur until a reflected wave has had time to propagate to the position of the node. Students may be challenged to draw the interference pattern and to infer the boundary condition at the reflector. Naturally, it is possible to set up an ultrasound generator producing steady emission. However, one of our goals is to use readily available equipment, so we used an ultrasonic ranger from our elementary lab.

In addition, the oscilloscope display allows the demonstrator to measure the period of the waves, and their speed can be calculated from the interference experiment in the usual way. All that is necessary is that the wavelength be found from the distance (times two since the path change is twice the displacement of 


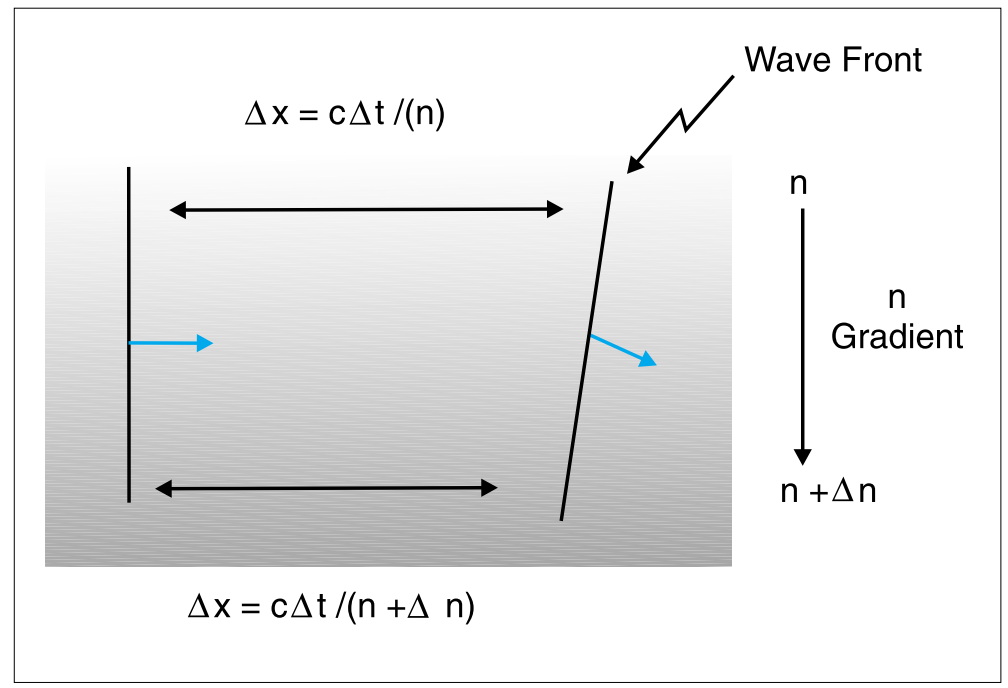

Fig. 5. Propagation of a wave front that is nominally parallel to a gradient in the index of refraction.

the reflector) between successive positions of the reflector providing constructive (or destructive) interference. Precision can be easily improved by measuring the displacement of the reflector for a number of antinodes (or nodes).

\section{A Simplified Quantitative Look at Laser Deflection}

It is possible to provide a mix of qualitative and simple quantitative discussion of how sound affects the propagation of the laser beam. Points to be noted would include the relationship between gas density and index of refraction, and the calculation of the ray deflection. A thorough treatment of the first of these draws on advanced physical optics, but we content ourselves with a qualitative discussion at the introductory level, emphasizing the conclusion that the denser the air, the slower the light wave travels through it. As to the deflection, it may seem as if we are headed towards a rather complex calculation. However, it turns out that the deflections are very slight and it is appropriate to treat the angle between the sound ray and the light ray as approximately constant throughout the region of interaction. Thus, the small deflection that occurs is assumed to leave the sound and light rays almost perpendicular for the purposes of calculating the deflection.

Consider a segment of a plane wave front, represented by a vertical line at time $t$, propagating horizontally from left to right; see Fig. 5 (where the deflection of the laser beam has been greatly exaggerated). We also assume there is a vertical gradient in the index of refraction. The index of refraction is $n+\Delta n$ at the bottom of the segment of wave front and it has the value $n$ at a distance $\Delta L$ above. Then the gradient is approximately $\Delta n / \Delta L$. In time $\Delta t$ the upper end of the wave front will move $\Delta x=c \Delta t / n$ while the lower end will move a slightly smaller amount, $\Delta x=$ $c \Delta t /(n+\Delta n)$. Disregarding quantities smaller than $\Delta t \Delta n$, the top of the line

\section{Appendix: Support Electronics}

We have followed the suggestion of the PSD manufacturer in building a circuit that uses divider and adder circuitry to produce a normalized output that is independent of the light spot intensity; see Fig. 6. The authors of Ref. 2 present a related, somewhat simpler circuit that does not provide a normalized output. However, they successfully use the data from it in a detailed analysis. Since the primary objective in this article is to present demonstration experiments of a qualitative nature, it may be wise to use their circuit.

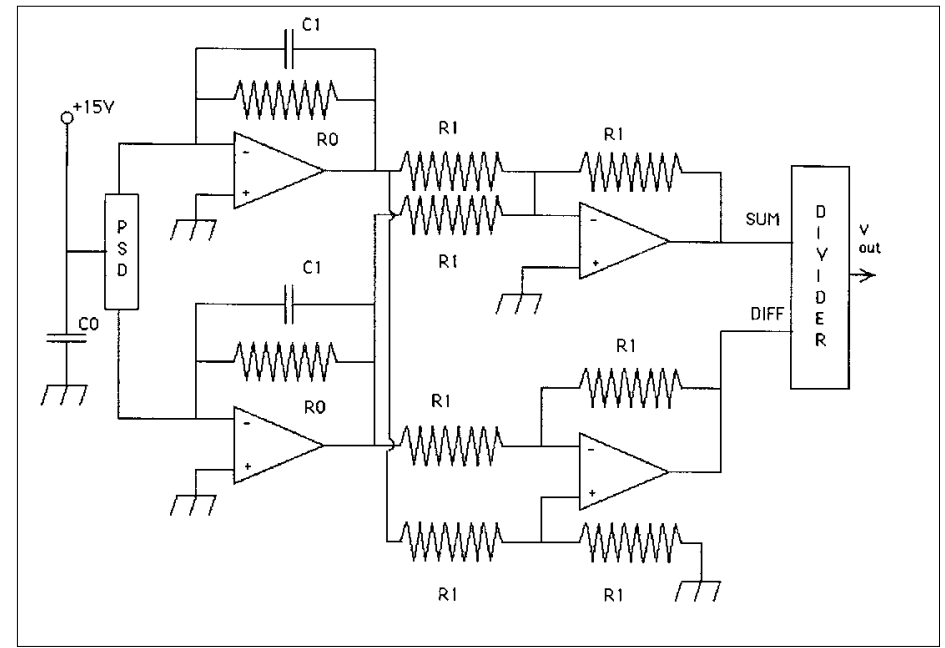

Fig. 6. Circuitry for the PSD. $\mathrm{C} 0=4.3 \mu \mathrm{F}, \mathrm{C} 1=50 \mathrm{pF}, \mathrm{R} 1=10 \mathrm{k} \Omega$, and $\mathrm{R} 0$ ranges from $10 \mathrm{k} \Omega$ to $1 \mathrm{M} \Omega$ depending on the amplification needed (e.g., we found 100 k $\Omega$ suitable). The op-amps were LM 307 N's and the divider was an AD533. Power was derived from an AD904 power unit. 
will have fallen behind the bottom by an amount $c \Delta t \Delta n / n^{2}$. This corresponds to a rotation of the wave front-and therefore of the ray-by an angle $\Delta \theta=(\Delta n / \Delta L)(\Delta x / n)$. Noting that the index of refraction of air is only slightly larger than 1 , we see that the deflection of the ray is proportional to the gradient, $\Delta n / \Delta L$, of the index of refraction and to the distance traveled.

\section{References}

1. Hamamatsu PSD \#S3932. Hamamatsu Corp., Bridgewater, NJ 08807. Presently $\$ 48$ each in small quantity orders (<10); 800-524 -0504.

2. C.L. Enloe, M.L. Brake, and T.E. Repetti, "Laser deflection by a spark," Am. J. Phys. 58, 400-403 (1990).

3. M. Minnaert, The Nature of Light and Color in the Open Air (Dover, New York, 1954), pp. 39-40.
4. R.P. Feynman, R.B. Leighton, and M. Sands, The Feynman Lectures on Physics, Volume I (California Institute of Technology, 1963), p. 26-5.

5. Vernier Software, 13979 Millikan Way, Beaverton, OR 97005-2886.

6. We were able to detect the effect in this case by performing a Fourier analysis on the PSD signal to overcome the signal-to-noise problem.

\section{TRICK OF THE TRADE}

\section{Diffraction by a Cat's Whisker}

The diffraction patterns produced by a single slit of width $w$ and a thin wire of the same width are exactly the same. This is called Babinet's principle and is based on the fact that the interference pattern produced by blocking out part of a wavefront depends only on the locations of the edges of the diffraction obstacle.

You are in the classroom and just about to derive the equation for the angular position of the first minimum for the diffraction pattern of a single slit of width $w: \lambda=w \sin \theta$. But first you want to show the phenomenon of single-slit diffraction and explore the variables with the students. Changing the wavelength is hard unless you have a set of red, green, and orange lasers. Showing how the angle depends on $w$ is easy-if you have a slit or a wire of variable width.

Nature provides us with ready-made wires of variable diameter in the form of cat whiskers. My two longhaired literary cats, Oliver Wendell Holmes and Emily Dickinson, shed fur and whiskers constantly; finding a dropped whisker is a regular occurrence at our house.

I taped one of Oliver's whiskers horizontally on a plate of glass at a height where the beam from a small red HeNe laser could strike it. Moving the plate sideways made the diffraction pattern expand as I moved toward the thin end of the whisker. On a screen $1.91 \mathrm{~m}$ distant, the diffraction minima for the thick part of the whisker were $0.50 \mathrm{~cm}$ apart, while toward the end they were $1.6 \mathrm{~cm}$ apart. For the small angles involved, the distance between adjacent minima (apart from the first ones on either side of the central maximum) are the same. Using $\lambda=633 \mathrm{~nm}$, the thick end of the whisker was thus $0.24 \mathrm{~mm}$ in diameter, and the thin end was $0.076 \mathrm{~mm}$ across. Of course, the thin end of the whisker tapers down to nothing, but the tape holding the whisker covered the end. 